\title{
Computational Characterization of the Dependence of Halide Perovskite Effective Masses on Chemical Composition and Structure
}

\author{
Negar Ashari-Astani, ${ }^{\dagger, \dagger}$ Simone Meloni, ${ }^{\dagger, \S \odot ~ A m i r ~ H e s a m ~ S a l a v a t i, ~}{ }^{\|, \perp}$ Giulia Palermo, ${ }^{\dagger, \#}$ \\ Michael Grätzel, ${ }^{\bigcirc}$ and Ursula Rothlisberger*,† \\ ${ }^{\dagger}$ Laboratory of Computational Chemistry and Biochemistry (LCBC), "Audiovisual Communications Laboratory (LCAV), and \\ OLaboratory of Photonics and Interfaces (LPI), École Polytechnique Fédérale de Lausanne, Lausanne, Switzerland CH-1015 \\ ${ }^{\S}$ Department of Mechanical and Aerospace Engineering, University of Rome "Sapienza", via Eudossiana 18, 00184 Roma, Italy \\ ${ }^{\ddagger}$ Department of Physics and ${ }^{\perp}$ Innovation Center (ICT), Sharif University of Technology, Tehran, Iran \\ \#Department of Chemistry and Biochemistry, University of California, San Diego, La Jolla, California 92093 United States
}

Supporting Information

\begin{abstract}
Effective masses are calculated for a large variety of perovskites of the form $\mathrm{ABX}_{3}$ differing in chemical composition $(\mathrm{A}=\mathrm{Na}, \mathrm{Li}, \mathrm{Cs} ; \mathrm{B}=\mathrm{Pb}, \mathrm{Sn} ; \mathrm{X}=$ $\mathrm{Cl}, \mathrm{Br}, \mathrm{I})$ and crystal structure. In addition, the effects of some defects and dopants are assessed. We show that the effective masses are highly correlated with the energies of the valence-band maximum, conduction-band minimum, and band gap. Using the $\mathbf{k} \cdot \mathbf{p}$ theory for the bottom of the conduction band and a tight-binding model for the top of the valence band, this trend can be rationalized in terms of the orbital overlap between halide and metal (B cation). Most of the compounds studied in this work are good charge-carrier transporters, where the effective masses of the $\mathrm{Pb}$ compounds $\left(0<m_{\mathrm{h}}^{*}<m_{\mathrm{e}}^{*}<1\right)$ are systematically larger than those of the

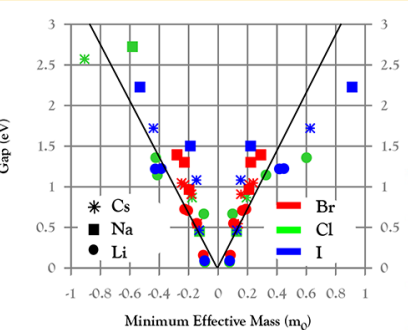

Minimum Effective Mass $\left(\mathrm{m}_{\mathrm{o}}\right)$

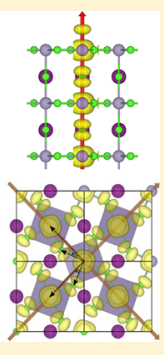
Sn-based compounds $\left(0<m_{\mathrm{h}}^{*} \approx m_{\mathrm{e}}^{*}<0.5\right)$. The effective masses show anisotropies depending on the crystal symmetry of the perovskite, whether orthorhombic, tetragonal, or cubic, with the highest anisotropy for the tetragonal phase (ca. 40\%). In general, the effective masses of the perovskites remain low for intrinsic or extrinsic defects, apart from some notable exceptions. Whereas some dopants, such as $\mathrm{Zn}(\mathrm{II})$, flatten the conduction-band edges $\left(m_{\mathrm{e}}^{*}=1.7 m_{0}\right)$ and introduce deep defect states, vacancies, more specifically $\mathrm{Pb}^{2+}$ vacancies, make the valence-band edge more shallow $\left(m_{\mathrm{h}}^{*}=0.9 m_{0}\right)$. From a device-performance point of view, introducing modifications that increase the orbital overlap [e.g., more cubic structures, larger halides, smaller (larger) monovalent cations in cubic (tetragonal/orthorhombic) structures] decreases the band gap and, with it, effective masses of the charge carriers.
\end{abstract}

\section{INTRODUCTION}

Twenty years after their first discovery as possible transistors, ${ }^{1}$ halide organic/inorganic perovskites (HOPs) with the composition $\mathrm{ABX}_{3}(\mathrm{~A}=$ organic or inorganic monovalent cation, $\mathrm{B}=$ bivalent cation, $\mathrm{X}=$ halide) have attracted a great deal of attention because of their breakthrough performance in third-generation solar cells. ${ }^{2-4}$ Efficiencies as high as $22.1 \%{ }^{5}$ have recently been reported for perovskite solar cells, and future efficiency increases up to $30 \%$, close to the ShockleyQueisser limit, seem feasible. ${ }^{6}$ Perovskites owe their superb performance to their high open-circuit voltages $\left(V_{\mathrm{oc}} \approx 1 \mathrm{~V}\right.$ for iodide perovskites and up to $\sim 1.5 \mathrm{~V}$ for bromide perovskites) ${ }^{7,8}$ long charge-carrier lifetime $(>15 \mu \mathrm{s}),{ }^{9,10}$ and low nonradiative carrier recombination rates (ca. $8 \times 10^{-12} \mathrm{~cm}^{3}$ $\left.\mathrm{s}^{-1}\right) .{ }^{11}$ The appropriate band gap of $\sim 1.65 \mathrm{eV}$ makes the classic methylammonium lead iodide perovskite $\left(\mathrm{CH}_{3} \mathrm{NH}_{3} \mathrm{PbI}_{3}\right)$ an excellent light harvester and, performance-wise, puts it in the class of highly efficient materials for thin-film solar cells, at a level comparable to that of $\mathrm{CdTe}$ and copper indium gallium (di)selenide (CIGS).

Hand in hand with experimental studies, computational investigations have been undertaken to shed light on the origin of the unique electronic properties of halide perovskites. Using density functional theory (DFT), the effects of halide and cation variations on the optical band gap and band structure have been investigated, along with the effects of temperature, the influence of crystal defects, steric effects, and the possible role of the perovskite $/ \mathrm{TiO}_{2}$ interface. ${ }^{6,12-17}$ Because of a fortuitous cancellation of spin-orbit and many-body effects, standard DFT calculations within the generalized gradient approximation (GGA) are able to predict the band gaps of lead halide perovskites in close agreement with experimental measurements. The energetics (i.e., the relative energy differences between various phases) are also well described at the GGA level. ${ }^{18}$ Even more relevant to the present work, using GW as a reference, Umari et al. showed that spin-orbit coupling (SOC) is crucial to the accurate determination of the band structures of halide perovskites and that DFT + SOC can provide an adequate description of band dispersion close to the

Received: May 22, 2017

Revised: August 3, 2017

Published: August 29, 2017 
valence-band maximum (VBM) and conduction-band minimum (CBM). ${ }^{19}$

In our previous work, ${ }^{16}$ we demonstrated how one can rationalize and predict the effects of variations in chemical composition and symmetry on the VBM and CBM energies of halide perovskites through their effects on two key parameters, the overlap between metal and halide orbitals and the effective charge of the divalent cation, determining the energy of these orbitals. In this work, we delve deeper into another equally important property of HOPs that affects the charge-carriertransport characteristics: the hole and electron effective masses. It is worth stressing that charge-carrier transport is also affected by the scattering of charge carriers by phonons, which is not discussed here.

In the field of semiconductors, the semiclassical model of electron dynamics, in which electrons and holes are assigned effective masses, $m_{\mathrm{h}}$ and $m_{\mathrm{e}}$, respectively, has been very successful. ${ }^{20}$ Retaining much of the simplicity of free-electron models, an effective-mass picture provides semiquantitative predictions for one of the terms determining the efficiency of charge-carrier transport. Recently, this model was applied to some HOP systems by different groups. ${ }^{21-31}$ In these works, the authors computed effective masses of single halide perovskite systems $^{22-24,29}$ or compared the masses of a limited number of systems differing in the type of monovalent ${ }^{27,30,31}$ or divalent $^{26,28}$ cation or halide. ${ }^{21,25}$ Despite the diversity of the applied approaches for calculating the effective masses, all of the reported values (except for a few abnormal and inappropriate values that we discuss in the Theory section) unanimously agree that the effective masses of both electrons and holes are in the range of good carrier transporters, in accordance with the experimental measurements of carrier mobility. ${ }^{32,33}$ The physical/chemical origin of this property, however, has remained an open question. In this work, we calculate $m_{\mathrm{h}}$ and $m_{\mathrm{e}}$ for a wide range of $\mathrm{Sn}$ - and $\mathrm{Pb}$-based perovskites that differ in the chemical nature of the monovalent cation $(\mathrm{Na}, \mathrm{Li}$, $\mathrm{Cs}$, and some organic molecular ions), the halide (I, Br, and $\mathrm{Cl}$ ), and the crystal symmetry (cubic, tetragonal, and orthorhombic). Indeed, the broad range of systems investigated here made it possible to better understand the dependence of the effective masses on the chemical and physical properties of halide perovskites.

The fact that we consider all-inorganic halide perovskites might appear to be in conflict with the trend of focusing on hybrid organic-inorganic systems. The reason for our choice is manifold. Hybrid perovskites, in particular, $\mathrm{CH}_{3} \mathrm{NH}_{3} \mathrm{PbI}_{3}$, have a limited stability, which has been attributed to the decomposition of methylammonium promoted by humidity. ${ }^{34}$ Thus, researchers are trying to replace or limit the content of organic cations by developing all-inorganic or mixed organic/ inorganic-cation halide perovskites. For example, recently, it was shown that $\alpha-\mathrm{CsPb}_{3}$ quantum dots are stable in ambient air, ${ }^{35}$ and this or similar materials are candidates for addressing the problem of the stability of hybrid perovskites. Other authors have also reported the synthesis of $\mathrm{CsPbI}_{3}$ perovskites. ${ }^{36,37}$ Indeed, the general interest in mixed cation and/or halide perovskites, which have shown high efficiency and enhanced stability thanks to the replacement of methylammonium and/or formamidinium by inorganic cations and iodine by bromine, ${ }^{18,38}$ and the addition of small monovalent cations ${ }^{39}$ calls for the investigation of a broad set of systems. Moreover, the research in recent years has shown that halide perovskites have potential technological applications beyond photovoltaics (e.g., lasing, light-emitting diodes, photodetection), for which optical properties different from those needed for solar cells are sought. Thus, other systems, such as $\mathrm{CsPbr}_{3}$, are of great interest. Finally, a technical question concerns the modeling of rotationally highly mobile methylammonium (and formamidinium) ion in static first-principles calculations. Experiments and simulations (see, e.g., refs 40-44) have shown that the residence time of methylammonium in metastable orientation states is on the picosecond time scale. Thus, a single configuration of methylammonium-based halide perovskites is not representative of the state of the system. Previous computational works ${ }^{13,42}$ showed that the electronic structures of the VBM and CBM, which determine the effective masses, are related to the monovalent cations through the effects of this ion on the $\mathrm{BX}_{3}^{-} 3 \mathrm{D}$ network. In this work, we use Cs-based perovskites, in particular, $\mathrm{CsPbI}_{3}$, also to mimic the average structure of $\mathrm{CH}_{3} \mathrm{NH}_{3} \mathrm{PbI}_{3}$. We note that, in simulations, 3D perovskite $\mathrm{CsPbI}_{3}$ is metastable and has a band gap similar to that of $\mathrm{CH}_{3} \mathrm{NH}_{3} \mathrm{PbI}_{3}$. ${ }^{16}$ It is worth mentioning that analogous approaches have been employed in other works (see, e.g., ref 45; also, the analogies between $\mathrm{CH}_{3} \mathrm{NH}_{3}{ }^{+}$and $\mathrm{Cs}^{+}$are briefly discussed in ref 46).

Anticipating our results, we found that, consistent with tightbinding and $\mathbf{k} \cdot \mathbf{p}$ theories, ${ }^{47}$ effective masses are strongly correlated with the $\mathrm{B} / n \mathrm{~s}-\mathrm{X} / \mathrm{mp}$ orbital overlap and band gap. ${ }^{16}$ Thus, one can tune the electron and hole effective masses by acting on those parameters affecting the orbital overlap. We also investigate the effects of intrinsic (vacancies) and extrinsic (doping) defects on the effective masses. We found that, although doping might be beneficial in view of increasing the concentration of free charge carriers, it turns out that, for example, $\mathrm{Zn}$ (II) doping can have some detrimental effects on carrier transport in some cases.

\section{THEORY}

In the semiclassical theory of transport, ${ }^{48}$ the effective masses of holes $\left(m_{\mathrm{h}}\right)$ and electrons $\left(m_{\mathrm{e}}\right)$ control the response of these particles to an electric field. Thus, $m_{\mathrm{h}}$ and $m_{\mathrm{e}}$, together with the scattering of charge carriers by phonons, are two key quantities determining the transport properties of charge carriers in perovskites. In 3D crystal systems, the effective mass tensors of holes and electrons, namely, $\hat{M}_{\mathrm{h}}$ and $\hat{M}_{\mathrm{e}}$, respectively, are related to the Hessian matrix of the energy at the VBM or CBM: $\hat{M}_{\mathrm{h} / \mathrm{e}}=\left(\hbar^{2} / 2\right) \hat{\epsilon}_{\mathrm{h} / \mathrm{e}}^{\prime \prime-1}$, where $\hat{\epsilon}^{\prime \prime}$ is the Hessian matrix of elements $\hat{\epsilon}_{i, j}^{\prime \prime}=\partial^{2} \epsilon(\mathbf{k}) / \partial k_{i} \partial k_{j}, \epsilon$ is the energy of the frontier orbitals of the valence and conduction bands, and $(\cdot)^{-1}$ denotes an inverse matrix. Effective masses are more conveniently computed and analyzed in the reference frame of the eigenvectors of the Hessian matrix. In this framework, $\hat{M}_{\mathrm{h} / \mathrm{e}}$ is a diagonal matrix of elements $m_{\alpha}=\hbar^{2} /\left(2 \epsilon_{\alpha}^{\prime \prime}\right)$, where $\epsilon_{\alpha}^{\prime \prime}$ is one of the eigenvalues of the Hessian matrix. Indeed, $m_{\mathrm{h} / \mathrm{e}, \alpha}$ is the effective mass for hole/electron transport along the direction of the corresponding eigenvector.

In this work, effective masses are obtained according to the following algorithm: First, the Hessian matrix is computed from a parabolic fitting of the energy of the frontier orbitals of the valence and conduction bands at a set of $k$-points around $k_{0}$, the $k$-point corresponding to the VBM or CBM: $\epsilon(\mathbf{k})=\epsilon\left(\mathbf{k}_{0}\right)+$ $1 /{ }_{2}\left(\mathbf{k}-\mathbf{k}_{0}\right)^{\mathrm{T}} \hat{\epsilon}^{\prime \prime}\left(\mathbf{k}-\mathbf{k}_{0}\right)$ [where $(\cdot)^{\mathrm{T}}$ denotes the transpose of the vector]. In particular, we use a $3 \times 3 \times 3$ grid of $k$-points with a spacing of $\Delta k$. The suitable value for $\Delta k$ is discussed below. Second, the Hessian matrix is diagonalized to yield the principal axes of charge-carrier transport (eigenvectors) and 
their corresponding eigenvalues. Finally, from the eigenvalues of the Hessian matrix the effective masses along these eigenvectors are obtained, as discussed in the previous paragraph. We note that we focus on a specific sub-band around its maximum/minimum, that is, a band of same band index $n$, and not on the curvature of the overall valence or conduction band. Thus, we are able to determine the hole/ electron effective masses also when the maximum/minimum is split because of SOC in locally polarized domains, which has been shown to be present in some halide perovskites (see, e.g., refs 49 and 50).

The above procedure requires suitable values for $\Delta k=\mid \mathbf{k}-$ $\mathbf{k}_{0}$ l. To tune and validate this parameter, we focus on the largely studied $\mathrm{MAPbI}_{3}$ system $\left(\mathrm{MA}=\mathrm{CH}_{3} \mathrm{NH}_{3}{ }^{+}\right) \cdot{ }^{21,23,24,32}$ In Table 1 , we report the average values of the effective masses for three

Table 1. Average Hole and Electron Masses, $\bar{m}_{\mathrm{h}}$ and $\bar{m}_{\mathrm{e}}$, Respectively, for $\mathrm{MAPbI}_{3}$ Using the Appropriate Grid Spacing $\Delta k^{\prime}$, Together with Two Inappropriate (Too Small and Too Large) Grid Spacing Values, $\Delta k$ and $\Delta k^{\prime \prime}$, Respectively, Resulting in Abnormal Values for the Effective Masses

\begin{tabular}{|c|c|c|c|}
\hline & $\bar{m}_{\mathrm{h}}$ & $\bar{m}_{\mathrm{e}}$ & $R^{2}$ \\
\hline$\Delta k=|\vec{a}| / 100$ & 0.05 & 0.35 & $0.67,0.87$ \\
\hline$\Delta k^{\prime}=|\vec{a}| / 400$ & 0.19 & 0.32 & $0.93,0.9^{a}$ \\
\hline$\Delta k^{\prime \prime}=|\vec{a}| / 800$ & 18.66 & 0.03 & $0.80,0.71$ \\
\hline
\end{tabular}

${ }^{a} \Delta k^{\prime}$ gives the best $R^{2}$ values, showing the significance of the grid spacing on the fitting procedure.

different values of $\Delta k:|\vec{a}| / 100,|\vec{a}| / 400$, and $|\vec{a}| / 800$, where $|\vec{a}|$ is the length of a reciprocal lattice vector. Thus, although the spacing of the $k$-point grid is the same in all directions for cubic structures, it differs between the axial and equatorial directions for tetragonal systems and changes in all three directions for orthorhombic structures. The suitable value of $\Delta k$ depends on how broad the energy dispersion curve is around $k_{0}$ : Broader curves are best fitted with coarser grids, and narrower curves require smoother grids. In fact, if the grid spans a space that is too wide, the second-order approximation to the energy is insufficient. On the contrary, if the space spanned is too narrow, then the change in the energy from the maximum of the valence band (minimum of the conduction band) is negligible, and the error in the estimation of the Hessian, and therefore in the effective masses, is large. The suitability of a value of $\Delta k$ is measured by the coefficient of determination $R^{2}$ of the parabolic approximation of the dispersion curve $k_{0}$, which, typically, must be $\geq 0.9$. Table 1 shows that the effects of the grid spacing on the estimation of the masses can be quite dramatic, with hole and electron masses reaching values as high as $18.7 m_{0}$ and as low as $0.03 m_{0}$, respectively (where $m_{0}$ is the electron rest mass).

The present analysis suggests that a possible explanation for the anomalous data reported in refs 25 and 51 for orthorhombic $\mathrm{MAPbI}_{3}\left(m_{\mathrm{e}}=11.979 m_{0}\right)$ and $\mathrm{MASnCl}_{3}\left(m_{\mathrm{e}}=\right.$ $\left.13.19 m_{0}\right)$ might be due to an improper calculation procedure. On the contrary, the results reported in Table 1 for $\Delta k=|\vec{a}| /$ 400 (i.e., at the maximum value of $R^{2}$ ) are in good agreement with experimental ${ }^{32}$ and previous theoretical ${ }^{19,23}$ results. It must be remarked that other explanations are also possible, for example, the lack of SOC in the calculations of refs 25 and 51. However, in our simulations, which we performed with and without SOC, we never observed a change of approximately 2 orders of magnitude associated with spin-orbit effects.

Before closing this section, it is worth mentioning that other authors have used a different approach to avoid the problem of determining a suitable value for $\Delta k$. Brivio et al. ${ }^{30}$ described the energy dispersion curve close to $k_{0}$ by combining the usual second-order expansion with an additional $k$-dependent function. Fitting the energy dispersion curve near $k_{0}$ along one specific direction in reciprocal space with this more elaborate function, they obtained $k$-dependent effective masses that, in the limit of $k \rightarrow k_{0}$, are consistent with those presented in this work. $R^{2}$ in the table is the coefficient of determination of the sub-band energy distribution for $m_{\mathrm{h}}^{*}$ (left) and $m_{\mathrm{e}}^{*}$ (right).

\section{COMPUTATIONAL SETUP}

GGA-DFT in the Perdew-Burke-Ernzerhof (PBE) formulation $^{52}$ is used to optimize the atomic configuration and lattice

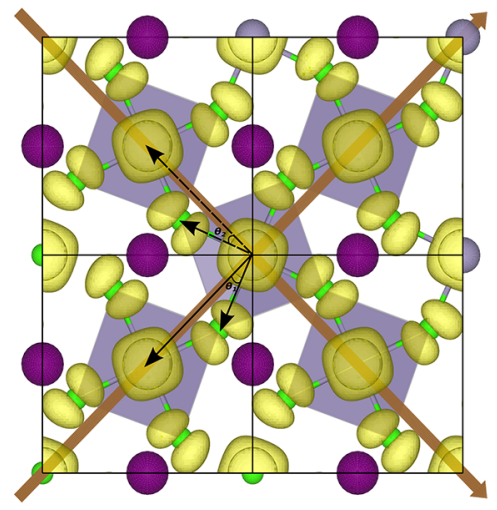

(a) Transversal principle axes.

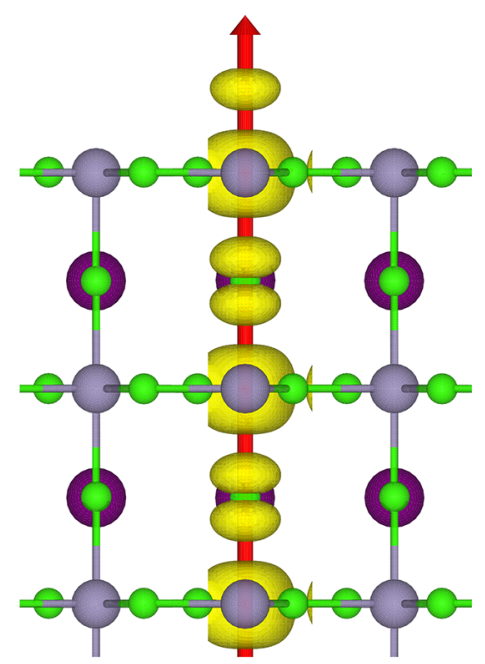

(b) Longitudinal principal axis.

Figure 1. (Left) Transverse and (right) longitudinal principal axes of hole transport (arrow) for the tetragonal systems. Also for cubic and orthorhombic systems, the principal axes are oriented along the $\mathrm{B}-\mathrm{B}$ directions. The gray polygons in the figure represent $\mathrm{BX}_{6}^{4-}$ units. 

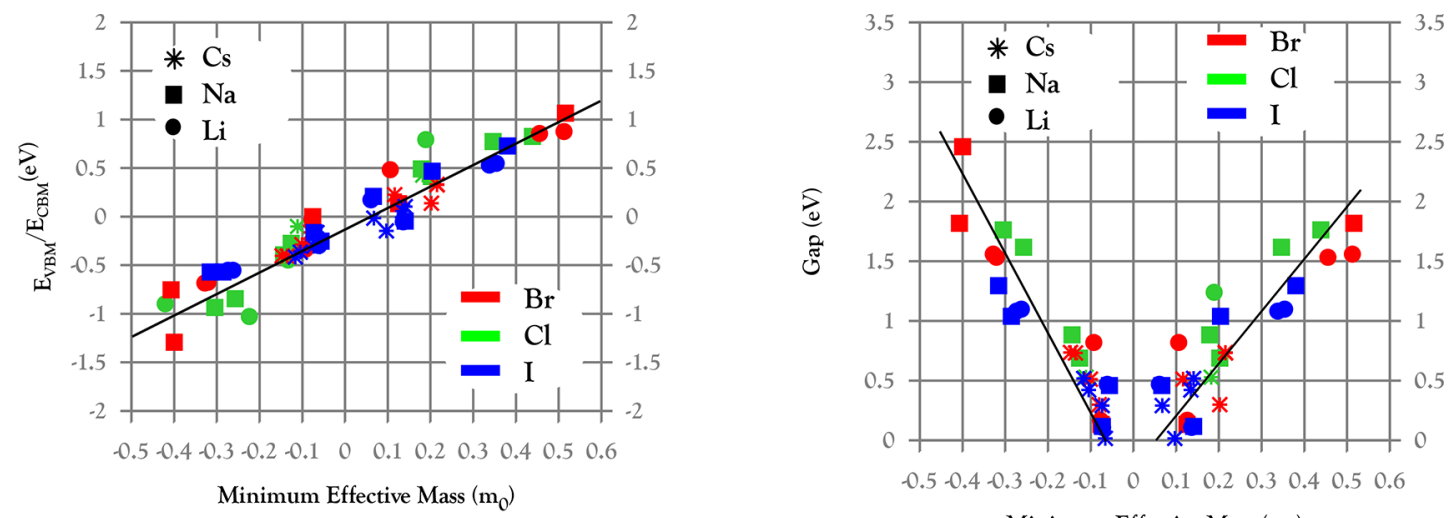

Minimum Effective Mass $\left(\mathrm{m}_{0}\right)$

Figure 2. (Left) Frontier orbital energies and (right) gaps versus minimum effective mass for Sn-based compounds $\mathrm{ASnX}$, $\mathrm{A}=\mathrm{Li}$, Na, and Cs (shown with diamonds, squares, and asterisks, respectively) and $\mathrm{X}=\mathrm{Cl}, \mathrm{Br}$, and $\mathrm{I}$ (shown in green, red, and blue, respectively) for different crystal structures.

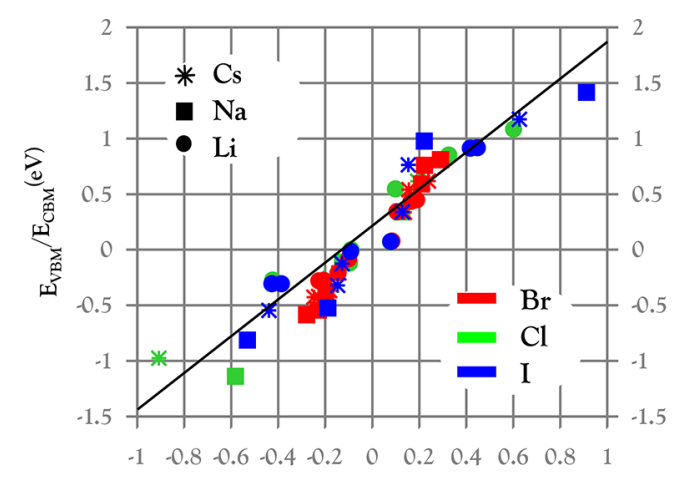

Minimum Effective Mass $\left(\mathrm{m}_{0}\right)$

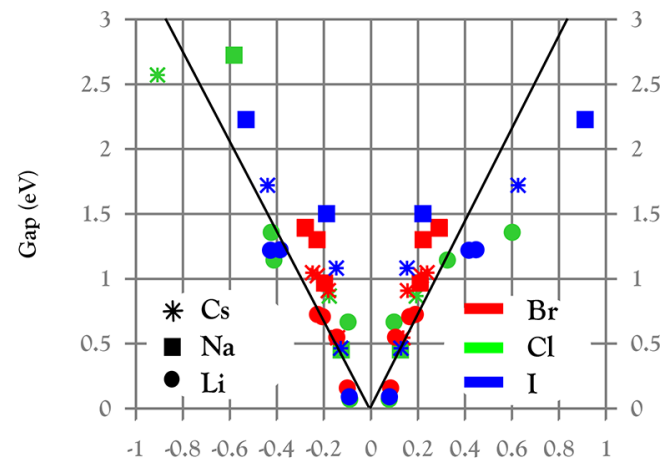

Minimum Effective Mass $\left(\mathrm{m}_{0}\right)$

Figure 3. (Left) Frontier orbital energies and (right) gaps versus minimum effective mass for Pb-based compounds $\mathrm{APbX}, \mathrm{A}=\mathrm{Li}, \mathrm{Na}$, and $\mathrm{Cs}$ (shown with diamonds, squares, and asterisks, respectively) and $\mathrm{X}=\mathrm{Cl}, \mathrm{Br}$, and I (shown in green, red, and blue, respectively) for different crystal structures.

parameters of all structures. Calculations are performed using the pw.x code of the Quantum Espresso package. ${ }^{53}$ Ultrasoft pseudopotentials are used to describe the interaction between the (semi)valence electrons and the nuclei and core electrons for all of the atoms. The Kohn-Sham orbitals and the total electronic density are expanded in a plane-wave basis with energy cutoffs of 40 and $280 \mathrm{Ry}$, respectively. The Brillouin zone is sampled with a $3 \times 3 \times 3$ or $4 \times 4 \times 4$ MonkhorstPack $k$-point grid $^{54}$ for cubic and tetragonal/orthorhombic structures, with supercells containing eight and four stoichiometric units, respectively. These values were chosen by checking the convergence of the total energy $\left(\sim 1 \times 10^{-3}\right.$ Ry/atom), band gap $(0.01 \mathrm{eV})$, and atomic forces $\left(\sim 1 \times 10^{-4}\right.$ $\mathrm{Ry} / \mathrm{au})$.

GGA-SOC calculations were performed on the perovskite systems to compute their band structures. The effective masses of holes and electrons were obtained by performing a quadratic fit of the $3 \mathrm{D}$ band structure at $k_{0}$, the $k$-point corresponding to the VBM and CBM. This required the calculation of the energies of frontier states of the valence and conduction bands on a $3 \times 3 \times 3$ grid of $k$-points centered at $k_{0}$ [see Figure $S 1$ in the Supporting Information (SI)]. The suitable number of grid points and grid spacing, $\Delta k$, were carefully chosen and tested system by system. The quadratic least-squares fit of the dispersion of the valence and conduction bands at $k_{0}$ was performed using the "Isqcurvefit" function of MATLAB 7.12. ${ }^{55}$ All of the fits present a value of the norm of the residual lower than $1.5 \times 10^{-9} \mathrm{eV}$, indicating a rather accurate parabolic approximation of the valence and conduction bands.

\section{RESULTS AND DISCUSSION}

We first focus on the analysis of the results for defect-free $\mathrm{Cs}^{+}$/ $\mathrm{Na}^{+} / \mathrm{Li}^{+}$lead and tin perovskite of $\mathrm{I}^{-} / \mathrm{Br}^{-} / \mathrm{Cl}^{-}$. All of these systems present low hole and electron effective masses close to the supercell $\Gamma$ point, with minimum values per system ranging from $\sim 0.1 m_{0}$ to $\sim 0.6 m_{0}$. The fact that the VBM and CBM occur near the $\Gamma$ point is due to the supercell used in the present work: this point folds back at the proper $k_{0}$ point for the unit cell of the given symmetry, for example, the $R$ point for cubic systems.

The principal axes of transport, that is, the eigenvectors of the effective mass tensor, are oriented along the $\mathrm{B}-\mathrm{B}$ directions (see Figure 1). These directions are all equivalent in the case of cubic systems, and thus, charge-carrier transport is isotropic (i.e., the masses along the three directions are the same). In the case of tetragonal systems, there are two degenerate principal axes of transport in the equatorial plane and one along the tetragonal axis. Finally, in orthorhombic systems, the three principal axes of transport, and the corresponding effective masses, are all different.

The dependence of the minimum hole and electron masses on the chemical composition (monovalent cation and halide within lead and tin perovskites) and crystal structure presents interesting trends. For a given chemical composition, the 


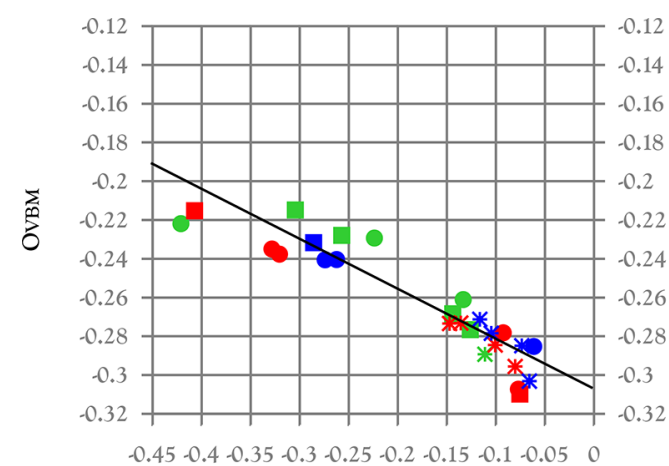

Minimum Effective Mass $\left(\mathrm{m}_{\mathrm{o}}\right)$

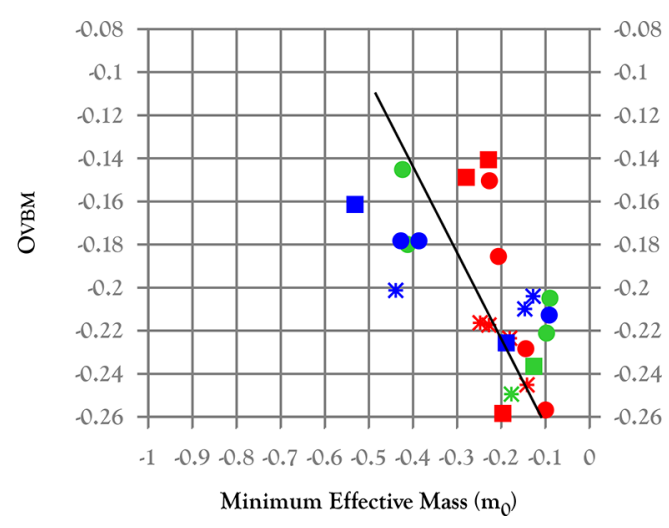

Figure 4. Antibonding overlap of the VBM orbitals $O_{\mathrm{VBM}}$ versus the minimum hole effective masses $m_{\mathrm{h}}^{*}$ for (left) tin-based and (right) lead-based compounds. $O_{\mathrm{VBM}}=\operatorname{Re}\left(\sum_{i \in \mathrm{X}-m \mathrm{p}} \sum_{j \in \mathrm{B}-m \mathrm{~s}} c_{\mathrm{VBM}, i}^{*} c_{\mathrm{VBM}, j} O_{i j}\right)$, where $O_{i j}=\int \mathrm{d} r \Phi_{i}^{*}(r) \Phi_{j}(r)$ is the overlap between pairs of X and B atomic orbitals and $c_{j}$ and $c_{i}$ are projection coefficients of the crystal orbitals onto the atomic orbitals.

effective masses increase along the series cubic $\rightarrow$ tetragonal $\rightarrow$ orthorhombic. If one considers the dependence on halides, keeping the types of mono- and bivalent cations and the crystal structure fixed, the masses grow along the sequence $\mathrm{I}^{-} \rightarrow \mathrm{Br}^{-}$ $\rightarrow \mathrm{Cl}^{-}$. The dependence on the type of monovalent cation is more complex and varies as a function of the crystal symmetry. For cubic systems, the masses grow along the sequence $\mathrm{Li}^{+} \rightarrow$ $\mathrm{Na}^{+} \rightarrow \mathrm{Cs}^{+}$, that is, they grow with the cation size. In tetragonal and orthorhombic systems, the masses grow in the opposite order: $\mathrm{Cs}^{+} \rightarrow \mathrm{Na}^{+} \rightarrow \mathrm{Li}^{+}$.

An analogous dependence on the chemical and physical characteristics of the halide perovskites has been observed for the VBM and CBM energies. ${ }^{16}$ This suggests a strong correlation between the minimum hole and electron masses $\left(m_{\mathrm{h}}^{*}\right.$ and $\left.m_{\mathrm{e}}^{*}\right)$ and the energy levels of the frontier orbitals of the valence and conduction bands. This correlation is shown in Figures 2 and 3 for lead and tin perovskites, respectively. In the panels on the left of these figures, the masses of holes and electrons as function of $\Delta E_{\mathrm{VBM}}$ and $\Delta E_{\mathrm{CBM}}$ are reported, where $\Delta E=E-E_{\min }$ and $E_{\min }$ is the minimum of the VBM or CBM among all of the systems considered. We remark that, in these and the following figures, the effective masses of holes are reported with a negative sign, so that the masses of both carriers can be plotted on the same chart. One can observe that the trend is almost linear with $\Delta E$ : the masses grow with the value of this parameter. Because $E_{\mathrm{VBM}}$ and $E_{\mathrm{CBM}}$ exhibit opposite trends with the composition and crystal symmetry (i.e., they concur in the widening or shrinking of the band gap $\left.E_{\mathrm{g}}=E_{\mathrm{CBM}}-E_{\mathrm{VBM}}\right), m_{\mathrm{h}}^{*}$ and $m_{\mathrm{e}}^{*}$ have a linear trend with the band gap as well.

The correlation between the hole and electron effective masses and the band gap in the direction observed in this work is consistent with the predictions of both the tight-binding (TB) and k $\cdot \mathbf{p}$ theories. ${ }^{47}$ Nevertheless, halide perovskite have some unconventional features that make the relationship between the properties of the material and its composition and structure less obvious and intuitive. As explained in ref 16, the VBM has a (antibonding) covalent character; thus, TB is well suited for interpreting the properties of this band. On the contrary, the CBM has a much less covalent character, and $\mathbf{k} \cdot \mathbf{p}$ theory works better in describing this case. This suggests that a single theory will not be adequate for interpreting the computational results and linking them to the characteristics of the material, and one has to treat the cases separately.
Concerning $m_{\mathrm{h}}^{*}$, the dependence of the effective mass on the energy of the corresponding band can be explained as follows: Within TB, the curvature of the VBM grows with the overlap $O_{\mathrm{VBM}}$ between the atomic orbitals contributing to the band (the $\mathrm{s}$ orbitals of $\mathrm{Sn} / \mathrm{Pb}$ and the $\mathrm{p}$ orbitals of $\mathrm{X}$ atoms in $\mathrm{the}$ present case). [Note $\mathrm{that}$ $O_{\mathrm{VBM}}=\operatorname{Re}\left(\sum_{i \in \mathrm{X}-m \mathrm{p}} \sum_{j \in \mathrm{B}-m \mathrm{~s}} c_{\mathrm{VBM}, i}^{*} c_{\mathrm{VBM}, j} O_{i j}\right), \quad$ w h e r e $O_{i j}=\int \mathrm{d} r \Phi_{i}^{*}(r) \Phi_{j}(r)$ and $c_{\mathrm{VBM}, i}$ and $c_{\mathrm{VBM}, j}$ are the contributions of atomic orbitals $i$ and $j$, respectively, to the VBM.] In practice, the higher the overlap and the higher the curvature of the band, the lower associated effective mass (see Figure 4). At the same time, the orbital overlap also determines the energy of the VBM, which explains the correlation between $m_{\mathrm{h}}^{*}$ and $E_{\mathrm{VBM}}$ (Figures 2 and 3 ).

Concerning $m_{\mathrm{e}}^{*}$, as mentioned above, $\mathbf{k} \cdot \mathbf{p}$ theory is more suitable for explaining the computational results relative to the conduction band and, thus, the electron effective masses. $\mathbf{k} \cdot \mathbf{p}$ theory is a perturbative approach to the calculation of the energy of the band at a $\mathbf{k}$ point in the neighborhood of the CBM

$$
E_{k}^{\mathrm{CB}}=E_{k_{0}}^{\mathrm{CB}}+\frac{\hbar^{2} k^{2}}{2 m}+\frac{\hbar^{2}}{m^{2}} \sum_{l} \frac{\left|\mathbf{k} \cdot\left\langle u_{k_{0}}^{l}|\mathbf{p}| u_{k_{0}}^{\mathrm{CB}}\right\rangle\right|^{2}}{E_{k_{0}}^{\mathrm{CB}}-E_{k_{0}}^{l}}
$$

For the sake of simplicity, we report here the form without SOC. Nevertheless, this form is sufficient to explain the dependence of $m_{\mathrm{e}}^{*}$ on the composition and crystal structure of the material. $m_{\mathrm{e}}^{*}$ is related to the second-order perturbative term, that is, it depends on the transition moment integral, $\left\langle u_{k_{0}}^{l}\right|$ $\mathbf{p}\left|u_{k_{0}}^{\mathrm{CB}}\right\rangle$, and the energy difference between the CB at $k_{0}$ and the other bands at the same point, $E_{k_{0}}^{\mathrm{CB}}-E_{k_{0}}^{m}$. In practice, relevant contributions to $E_{k}^{\mathrm{CB}}$ come from bands of suitable symmetry ( $<$ $\left.u_{k_{0}}^{l}|\mathbf{p}| u_{k_{0}}^{\mathrm{CB}}\right\rangle \neq 0$ ) that are close in energy to the CBM (small $E_{k_{0}}^{\mathrm{CB}}$ $\left.-E_{k_{0}}^{m}\right)$. Because the transition moment integral vanishes for most of the conduction bands (see Tables S1 and S2 in the SI), in the present case, the major contribution comes from the VBM. Thus, $m_{\mathrm{e}}^{*}$ depends on the band gap and, as we showed in ref 16 , this is correlated to $O_{\mathrm{VBM}}$.

In summary, modifications of the chemical composition and crystal structure are effective ways of controlling the orbital overlap and, through it, $m_{\mathrm{h}}^{*}$ and $m_{\mathrm{e}}^{*}$. For example, cubic perovskites, with linear $\mathrm{B}-\mathrm{X}-\mathrm{B}$ bonds, have maximum overlap, 


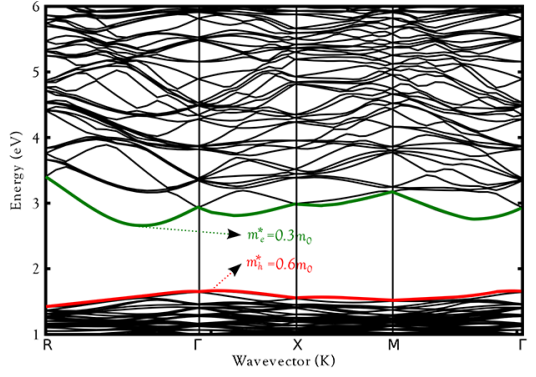

(a) $\mathrm{NH}_{4} \mathrm{PbI}_{3}$

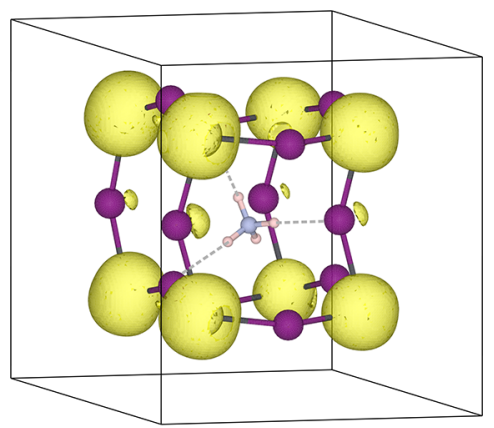

(c) $\mathrm{CBM}-\mathrm{NH}_{4} \mathrm{PbI}_{3}$

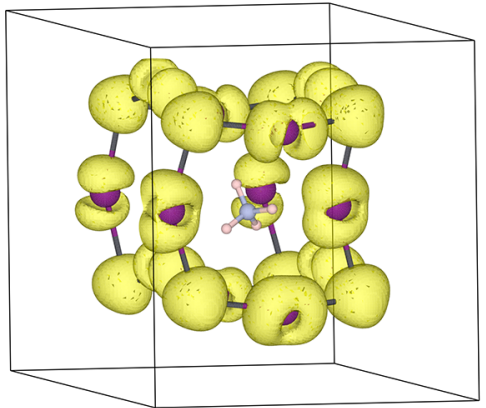

(e) $\mathrm{VBM}-\mathrm{NH}_{4} \mathrm{PbI}_{3}$

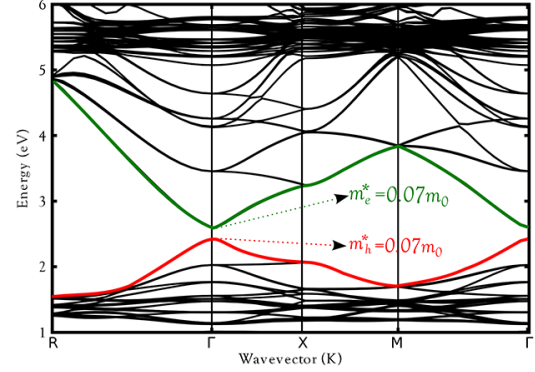

(b) $\mathrm{PH}_{4} \mathrm{PbI}_{3}$

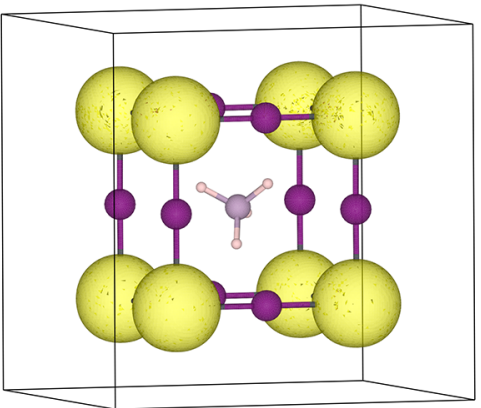

(d) $\mathrm{CBM}-\mathrm{PH}_{4} \mathrm{PbI}_{3}$

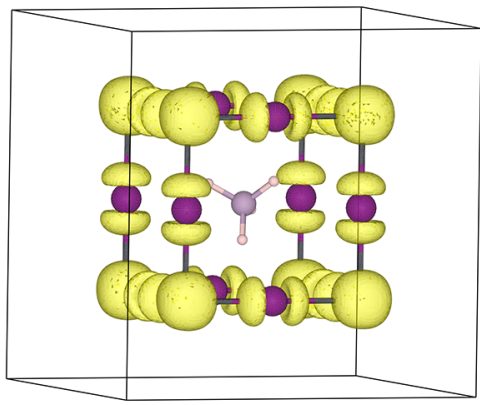

(f) VBM-PH $\mathrm{H}_{4} \mathrm{PbI}_{3}$

Figure 5. Effects of hydrogen bonding on the effective masses. The distortion caused by hydrogen bonding widens the gap.

and as a consequence, they have lower masses with respect to the tetragonal and orthorhombic structures. The effect of halides is associated with the ratio between their ionic and covalent radii. The first contributes to the determination of the lattice size, and the second contributes to the orbital overlap (for a fixed interatomic distance). This ratio increases along the sequence $\mathrm{I}^{-} \rightarrow \mathrm{Br}^{-} \rightarrow \mathrm{Cl}^{-}$and, thus, $m_{\mathrm{h}}^{*}$ and $m_{\mathrm{e}}^{*}$ both decrease along the sequence. Finally, monovalent cations have a different effect depending on whether they are in cubic or tilted structures (tetragonal, orthorhombic). In the first case, they affect only the lattice size. Thus, smaller ions result in larger overlap and smaller effective masses. In tilted structures, on the contrary, the ionic size of the monovalent cation has twice the effect of affecting the lattice size and the linearity of $\mathrm{B}-\mathrm{X}-\mathrm{B}$ bonds, with the latter effect dominating the overlap. Thus, in tetragonal and orthorhombic structures, smaller monovalent cations reduce the overlap and increase $m_{\mathrm{h}}^{*}$ and $m_{\mathrm{e}}^{*}$.

Effects of Hydrogen Bonding. Organic cations can act as hydrogen-bond donors, engaging in hydrogen bonds with halide ions, possibly distorting the $\mathrm{BX}_{3}$ framework. An example of this effect occurs in $\mathrm{NH}_{4} \mathrm{PbI}_{3}$, in which the cubic structure is significantly distorted. This results in a significant change in the band structure, with the CBM moving toward the center of the line connecting the $R$ and $\Gamma$ high-symmetry points of the reciprocal lattice (see Figure $5 \mathrm{e}, \mathrm{c}$ ). The distortion of the crystal structure results in a significant reduction of the orbital overlap, ${ }^{16}$ which, as expected, induces the flattening of the VBM with respect to the case of a much weaker hydrogen-bond donor, such as $\mathrm{PH}_{4} \mathrm{PbI}_{3}$ (Figure 5f,d). This results in an increase in the effective masses by an order of magnitude in going from $\mathrm{PH}_{4} \mathrm{PbI}_{3}$ to $\mathrm{NH}_{4} \mathrm{PbI}_{3}$ (yet smaller than $1.5 m_{0}$ ).

It must be noted that the computational setup described in the corresponding section, which is analogous to the one used in the vast majority of recent literature in the field, ${ }^{46}$ does not take into account van der Waals (vdW) interactions, which might be important in perovskites, ${ }^{56}$ especially for systems involving hydrogen bonding. Indeed, the relevance of $\mathrm{vdW}$ interactions in halide perovskites has been debated in the literature, with authors suggesting that related corrections compensate for other limitations of GGA-DFT ${ }^{46}$ or call for a more physically sound justification of the relevance of dispersion forces in this class of systems. ${ }^{57}$ Here, we take the pragmatic approach of testing the effects of $\mathrm{vdW}$ interactions for hydrogen-bonding systems $\left(\mathrm{NH}_{4} \mathrm{PbI}_{3}\right.$ and $\left.\mathrm{PH}_{4} \mathrm{PbI}_{3}\right)$. We optimized the atomic structure, including the periodic cell, and computed the band structure with three empirical (DFT-D $2^{58}$ ) 


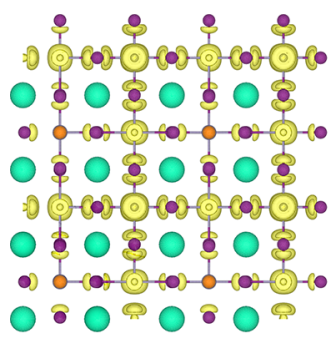

(a) VBM

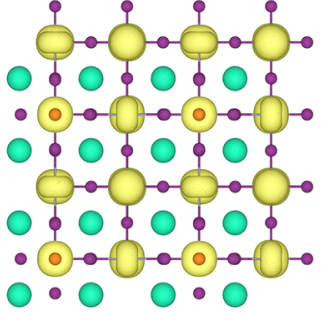

(b) Independent band

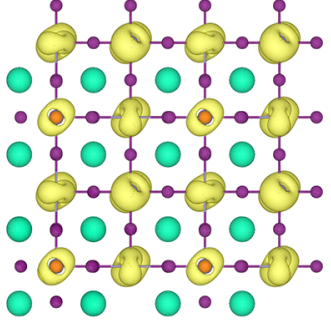

(c) CBM

Figure 6. Effects of $\mathrm{Zn}$ (II) dopant on the orbitals of the band edges.

and nonlocal (vdW-DF ${ }^{59}$ and vdW-DF2 $2^{60}$ ) methods that take dispersion interactions into account. In Figure S7 in the SI, we report the band structures of the $\mathrm{VBM}$ and $\mathrm{CBM}$ of $\mathrm{NH}_{4} \mathrm{PbI}_{3}$ and $\mathrm{PH}_{4} \mathrm{PbI}_{3}$ obtained by the various methods. We found relatively small effects on the structural characteristics of $\mathrm{PH}_{4} \mathrm{PbI}_{3}$, with a change in the lattice parameters of $\leq 1 \%$ and a variation in the hole/electron effective masses of $\leq 0.02 m_{0}$. For $\mathrm{NH}_{4} \mathrm{PbI}_{3}$, the change in the structural characteristics was again small, with a variation in the lattice parameters of $<2 \%$. The variation in $m_{\mathrm{e}}^{*}$ was also small, of the same order of magnitude as that observed for $\mathrm{PH}_{4} \mathrm{PbI}_{3}$. In contrast, the position of the VBM and its dispersion changed significantly. In particular, the curvature of the VBM was further reduced, and $m_{\mathrm{h}}^{*}$ increased to $\sim 1.5 m_{0}$, reinforcing our conclusion that strong hydrogen bonding can increase the hole effective mass and, thus, decrease the performance of the material.

Effects of Dopants and Defects. The results discussed in the previous section concern perfect halide perovskite structures. However, materials used in devices contain (intrinsic) defects, especially in the case of perovskites produced by liquid processing; charged vacancy defects are estimated to exceed $0.4 \%$ at room temperature. ${ }^{61}$ Moreover, the addition of dopants results in the formation of extrinsic defects. Defects can affect charge-carrier masses in many ways. For example, they can introduce deep trap states that favor recombination and reduce the carrier lifetime. However, it has been shown that the most common intrinsic defects do not introduce these types of states in halide perovskites (see, e.g., refs 27,62 , and 63), resulting in a very low concentration of carrier traps. ${ }^{64}$ Less investigated are the effects of defects on the valence and conduction bands and, therefore, on the effective masses. In the following discussion, we consider the effects of $\mathrm{Cs}, \mathrm{Pb}$, and $\mathrm{I}$ vancancies and $\mathrm{Zn}$ and $\mathrm{Sr}$ doping on the effective masses of $\mathrm{CsSnI}_{3}$.

The presence of defects breaks the translational symmetry of ideal crystals. Nevertheless, first-principles calculations based on periodic systems have been shown to work well in investigations of the electronic structure of these types of systems. ${ }^{64,65}$ Thus, the effects of defects on effective masses have been studied using the same approach as described in the Theory section.

A final remark is necessary before proceeding with presenting our results. It is known that the GGA-DFT approach employed in this and most other computational works on defects in halide perovkites ${ }^{23,62,66}$ has potential shortcomings. ${ }^{67}$ However, the long lifetimes of carriers in halide perovskites suggest that intrinsic defects do not introduce deep states, and the present simulation setup is expected to encounter fewer severe problems in this case. Indeed, in the absence of defects, SOC-GGA-DFT calculations give effective masses in quantitative agreement with SOC-Hybrid-DFT ${ }^{68}$ and SOC $-\mathrm{GW}^{19}$ calculations and experimental data. ${ }^{32}$ More recently, GGA-PBE calculations were employed to successfully interpret experimental data on the annihilation of Frenkel defects in $\mathrm{CH}_{3} \mathrm{NH}_{3} \mathrm{PbI}_{3}$. ${ }^{69}$ Finally, in his work on defects in $\mathrm{CH}_{3} \mathrm{NH}_{3} \mathrm{PbI}_{3}, \mathrm{Du}^{23}$ remarked that "PBE calculations [inclusive of SOC] provide sufficiently accurate results on forces (near equilibrium), structures, and band dispersion". These literature data make us confident that our setup is adequate for describing the (qualitative) effects of defects on the crystal band structure.

Anticipating our results, we remark that $\mathrm{V}_{\mathrm{Cs}}^{\prime}$ and $\mathrm{V}_{\mathrm{I}}^{\bullet}$ vacancies have minimal effects on the effective masses. On the contrary, $\mathrm{V}_{\mathrm{Pb}}^{\prime \prime}, \mathrm{Zn}_{\mathrm{Sn}}^{\times}$, and $\mathrm{Sr}_{\mathrm{Sr}}^{\times}$significantly increase $m_{\mathrm{h}}^{*}$ and $m_{\mathrm{e}}^{*}$.

Effects of Dopants: $\operatorname{CsSn}_{x} D_{1-x} I_{3}, D=Z n(I I), \operatorname{Sr}(I I)$. As a first scenario for doping, we substituted $\mathrm{Sn}$ with $\mathrm{Zn}$ and $\mathrm{Sr}$ at an 8:1 ratio. Both of these dopants widen the band gap (by 0.7 and 0.3 $\mathrm{eV}$ for $\mathrm{Zn}$ and $\mathrm{Sr}$, respectively) and split some degenerate states by breaking the symmetry (point $\mathrm{X}$ in Figure S3 in the SI). Whereas the main features of the band structure of the parent compound, $\mathrm{CsSnI}_{3}$, are preserved in the Sr-doped system, the band structure of the Zn-doped system changes drastically. Zinc doping creates an empty additional localized level within the band gap (n-like doping) and considerably changes the shape of the CBM. This new level is composed of Sn p and I s orbitals, along with a small contribution of $\mathrm{Zn}$ p orbitals. Being localized, this state does not contribute to the carrier transport.

The substitutional dopants $\mathrm{Zn}$ and $\mathrm{Sr}$ affect the electronic structures of the VBM and CBM. For instance, the presence of $\mathrm{Zn}$ suppresses the orbital contribution of the neighboring iodide ions, an effect that can be seen in the reduction of the electron density in the vicinity of $\mathrm{Zn}$ (II) (Figure 6). Despite this effect, the curvature of the VB at its maximum is large for both dopants, with average hole effective masses of $\sim 0.05 m_{0}$. For electron carriers, in contrast to strontium doping, $\mathrm{Zn}$ doping significanly affects the curvature of the CBM, giving rise to higher effective electron masses, $m_{\mathrm{e}}^{*}=1.7 m_{0}$.

Vacancy Defects (Cs, Pb, axial, and equatorial I) in $\mathrm{CsPbl}_{3}$. Early studies on the defect physics of halide perovskites showed that, despite their possible abundance, ${ }^{61}$ the dominant intrinsic defects create only shallow levels that are not too detrimental for the transport properties in this class of materials ${ }^{62}$ (see ref 70 for an exhaustive review on the physics and chemistry of defects in perovskites). However, the effects of simple point defects, namely, vacancies, on the valence and conduction bands of halide perovskites have not been considered. Here, we focus on $\mathrm{CsPbI}_{3}$ as a prototypical system for investigating this phenomenon.

The band structures of tetragonal $\mathrm{CsPbI}_{3}$ with different vacancies, namely, $\mathrm{V}_{\mathrm{C} s}^{\prime}, \mathrm{V}_{\mathrm{Pb}}^{\prime \prime}$, and $\mathrm{V}_{\mathrm{I}}^{\bullet}$ (equatorial and axial), suggest that the effective masses of holes and electrons have a 
low sensitivity to vacancies (Figure S5 in the SI). Except for an expected splitting of degenerate states (because of the broken symmetry), only $\mathrm{Pb}$ vacancies seem to have a non-negligible effect on the masses (mostly on $m_{\mathrm{h}}^{*}$ ). $\mathrm{Pb}$ vacancies flatten the VBM, resulting in relatively heavy holes: $m_{\mathrm{h}}^{*}=0.9 m_{0}$. This result might seem surprising, considering that $\mathrm{Pb}$ makes a relatively small contribution to this orbital. Indeed, the present results suggest that the electronic structure of perovskites is more complex than expected and reported in the literature. The sizable increase in $m_{\mathrm{h}}^{*}$ is due to direct and indirect effects of $\mathrm{V}_{\mathrm{Pb}}^{\prime \prime}$. Lead vacancies reduce the contribution of the $\mathrm{s} \mathrm{Pb}$ atomic orbital of the vacant atom to the VBM. This, in turn, affects the contribution of equatorial iodide $\mathrm{p}$ orbitals to the same band (Figure 7). This combined effect results in a significant decrease

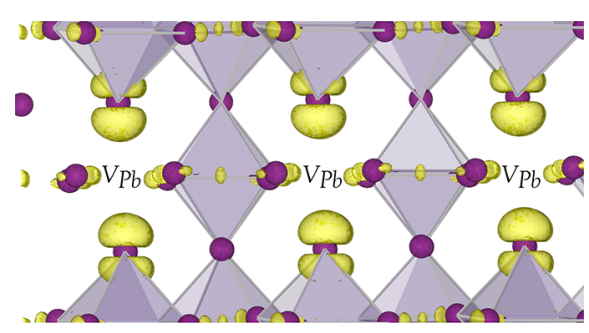

Figure 7. $\mathrm{Pb}$ vacancy: depletion of equatorial iodide p-orbital contributions around the vacancy that significantly affects the VBM.

of the overlap and a corresponding increase of the hole mass according to the same mechanism as discussed for defect-free systems.

To validate our calculations and confirm that our conclusions were not affected by significant finite-size effects, we repeated our calculations with a $2 \times 2$ simulation box, containing as many as 159 atoms. Effective masses computed for these larger systems showed negligible differences from those computed for the original systems.

\section{SUMMARY}

We performed first-principles calculations on a large set of halide perovskites with the aim of calculating their holes and electrons effective masses. Effective masses are strongly correlated with the energies of the VBM, CBM, and band gap. This can be explained by means of the TB and $\mathbf{k} \cdot \mathbf{p}$ theories for the valence and conduction bands, respectively. We also investigated the effects of intrinsic and extrinsic defects on the effective masses. Substitutional $\mathrm{Zn}^{2+}$ adds a localized state in the gap and flattens the band edges, especially the $\mathrm{CB}$ edge, resulting in higher effective masses. $\mathrm{Pb}^{2+}$ vacancies reduce the antibonding atomic orbital overlap, resulting in a reduction of the curvature of the $\mathrm{VB}$ and an increase of the hole mass. The other defects have minor effects on the effective masses.

\section{ASSOCIATED CONTENT}

\section{S Supporting Information}

The Supporting Information is available free of charge on the ACS Publications website at DOI: 10.1021/acs.jpcc.7b04898.

Some details of the procedure for the calculation of effective masses, symmetries of the bands in pure GGA and GGA + SOC of $\mathrm{CsSnI}_{3}$, and band structures of all of the systems investigated in this work (PDF)

\section{AUTHOR INFORMATION}

\section{Corresponding Author}

*E-mail: ursula.roethlisberger@epfl.ch.

ORCID

Simone Meloni: 0000-0002-3925-3799

Michael Grätzel: 0000-0002-0068-0195

\section{Notes}

The authors declare no competing financial interest.

\section{ACKNOWLEDGMENTS}

U.R. acknowledges funding from the Swiss National Science Foundation through individual Grant 200020-146645, NCCRs MUST and MARVEL, and support from the Swiss National Computing Center (CSCS), the CADMOS project, and NRP70. We also acknowledge PRACE for awarding us access to resource SuperMUC based in Germany at LeibnizRechenzentrum.

\section{REFERENCES}

(1) Mathews, S.; Ramesh, R.; Venkatesan, T.; Benedetto, J. Ferroelectric Field Effect Transistor Based on Epitaxial Perovskite Heterostructures. Science 1997, 276, 238-240.

(2) Mitzi, D. B. Synthesis, Structure, and Properties of OrganicInorganic Perovskites and Related Materials; Progress in Inorganic Chemistry; John Wiley \& Sons, Inc.: New York, 2007; Vol. 48, pp 1121.

(3) Tanaka, K.; Takahashi, T.; Kondo, T.; Umeda, K.; Ema, K.; Umebayashi, T.; Asai, K.; Uchida, K.; Miura, N. Electronic and Excitonic Structures of Inorganic-Organic Perovskite-Type QuantumWell Crystal $\left(\mathrm{C}_{4} \mathrm{H}_{9} \mathrm{NH}_{3}\right)_{2} \mathrm{PbBr}_{4}$. Jpn. J. Appl. Phys. 2005, 44, 5923.

(4) Snaith, H. J. Perovskites: the Emergence of a New Era for LowCost, High-Efficiency Solar Cells. J. Phys. Chem. Lett. 2013, 4, 36233630.

(5) Yang, W. S.; Park, B. W.; Jung, E. H.; Jeon, N. J.; Kim, Y. C.; Lee, D. U.; Shin, S. S.; Seo, J.; Kim, E. K.; Noh, J. H.; Seok, S. I. Iodide management in formamidinium-lead-halide-based perovskite layers for efficient solar cells. Science 2017, 356 (6345), 1376-1379.

(6) Grånäs, O.; Vinichenko, D.; Kaxiras, E. Establishing the Limits of Efficiency of Perovskite Solar Cells from First Principles Modeling. Sci. Rep. 2016, 6, 36108.

(7) Kulbak, M.; Cahen, D.; Hodes, G. How Important is the Organic Part of Lead Halide Perovskite Photovoltaic Cells? Efficient CsPbBr Cells. J. Phys. Chem. Lett. 2015, 6, 2452-2456.

(8) Edri, E.; Kirmayer, S.; Kulbak, M.; Hodes, G.; Cahen, D. Chloride Inclusion and Hole Transport Material Doping to Improve Methyl Ammonium Lead Bromide Perovskite-Based High Open-Circuit Voltage Solar Cells. J. Phys. Chem. Lett. 2014, 5, 429-433.

(9) Bi, Y.; Hutter, E. M.; Fang, Y.; Dong, Q.; Huang, J.; Savenije, T. J. Charge Carrier Lifetimes Exceeding $15 \mu \mathrm{s}$ in Methylammonium Lead Iodide Single Crystals. J. Phys. Chem. Lett. 2016, 7, 923-928.

(10) Stranks, S. D.; Eperon, G. E.; Grancini, G.; Menelaou, C.; Alcocer, M. J.; Leijtens, T.; Herz, L. M.; Petrozza, A.; Snaith, H. J. Electron-Hole Diffusion Lengths Exceeding 1 Micrometer in an Organometal Trihalide Perovskite Absorber. Science 2013, 342, 341344 .

(11) Stranks, S. D.; Burlakov, V. M.; Leijtens, T.; Ball, J. M.; Goriely, A.; Snaith, H. J. Recombination Kinetics in Organic-Inorganic Perovskites: Excitons, Free Charge, and Subgap States. Phys. Rev. Appl. 2014, 2, 034007.

(12) Mosconi, E.; Umari, P.; De Angelis, F. Electronic and Optical Properties of Mixed Sn-Pb Organohalide Perovskites: a First Principles Investigation. J. Mater. Chem. A 2015, 3, 9208-9215.

(13) Filip, M. R.; Eperon, G. E.; Snaith, H. J.; Giustino, F. Steric Engineering of Metal-Halide Perovskites with Tunable Optical Band Gaps. Nat. Commun. 2014, 5, 5757. 
(14) Even, J.; Pedesseau, L.; Katan, C.; Kepenekian, M.; Lauret, J.-S.; Sapori, D.; Deleporte, E. A Solid State Physics Perspective on Hybrid Perovskite Semiconductors. J. Phys. Chem. C 2015, 119, 10161-10177.

(15) Butler, K. T.; Frost, J. M.; Walsh, A. Band Alignment of the Hybrid Halide Perovskites $\mathrm{CH}_{3} \mathrm{NH}_{3} \mathrm{PbCl}_{3}, \mathrm{CH}_{3} \mathrm{NH}_{3} \mathrm{PbBr}_{3}$ and $\mathrm{CH}_{3} \mathrm{NH}_{3} \mathrm{PbI}_{3}$. Mater. Horiz. 2015, 2, 228-231.

(16) Meloni, S.; Palermo, G.; Ashari-Astani, N.; Grätzel, M.; Rothlisberger, U. Valence and Conduction Band Tuning in Halide Perovskites for Solar Cell Applications. J. Mater. Chem. A 2016, 4, 15997-16002.

(17) Dar, M. I.; Jacopin, G.; Meloni, S.; Mattoni, A.; Arora, N.; Boziki, A.; Zakeeruddin, S. M.; Rothlisberger, U.; Grätzel, M. Origin of Unusual Bandgap Shift and Dual Emission in Organic-Inorganic Lead Halide Perovskites. Sci. Adv. 2016, 2, e1601156.

(18) Yi, C.; Luo, J.; Meloni, S.; Boziki, A.; Ashari-Astani, N.; Grätzel, C.; Zakeeruddin, S. M.; Röthlisberger, U.; Grätzel, M. Entropic Stabilization of Mixed A-Cation $\mathrm{ABX}_{3}$ Metal Halide Perovskites for High Performance Perovskite Solar Cells. Energy Environ. Sci. 2016, 9, 656-662.

(19) Umari, P.; Mosconi, E.; De Angelis, F. Relativistic GW Calculations on $\mathrm{CH}_{3} \mathrm{NH}_{3} \mathrm{PbI}_{3}$ and $\mathrm{CH}_{3} \mathrm{NH}_{3} \mathrm{SnI}_{3}$ Perovskites for Solar Cell Applications. Sci. Rep. 2015, 4, 4467.

(20) Vurgaftman, I.; Meyer, J. R.; Ram-Mohan, L. R. Band Parameters for III-V Compound Semiconductors and their Alloys. J. Appl. Phys. 2001, 89, 5815-5875.

(21) Chang, Y. H.; Park, C. H.; Matsuishi, K. First-Principles Study of the Structural and the Electronic Properties of the Lead-Halide-Based Inorganic-Organic Perovskites $\left(\mathrm{CH}_{3} \mathrm{NH}_{3}\right) \mathrm{PbX}_{3}$ and $\mathrm{CsPbX}(\mathrm{X}=\mathrm{Cl}$, Br, I). J. Korean Phys. Soc. 2004, 44, 889-893.

(22) Giorgi, G.; Fujisawa, J.-I.; Segawa, H.; Yamashita, K. Small Photocarrier Effective Masses Featuring Ambipolar Transport in Methylammonium Lead Iodide Perovskite: A Density Functional Analysis. J. Phys. Chem. Lett. 2013, 4, 4213-4216.

(23) Du, M. H. Efficient Carrier Transport in Halide Perovskites: Theoretical Perspectives. J. Mater. Chem. A 2014, 2, 9091-9098.

(24) Menéndez-Proupin, E.; Palacios, P.; Wahnón, P.; Conesa, J. SelfConsistent Relativistic Band Structure of the $\mathrm{CH}_{3} \mathrm{NH}_{3} \mathrm{PbI}_{3}$ Perovskite. Phys. Rev. B: Condens. Matter Mater. Phys. 2014, 90, 045207.

(25) Feng, J.; Xiao, B. Crystal Structures, Optical Properties, and Effective Mass Tensors of $\mathrm{CH}_{3} \mathrm{NH}_{3} \mathrm{PbX}_{3}(\mathrm{X}=\mathrm{I}$ and $\mathrm{Br})$ Phases Predicted from HSE06. J. Phys. Chem. Lett. 2014, 5, 1278-1282.

(26) Even, J.; Pedesseau, L.; Jancu, J.-M.; Katan, C. DFT and k.p Modelling of the Phase Transitions of Lead and Tin Halide Perovskites for Photovoltaic Cells. Phys. Status Solidi RRL 2014, 8, 31-35.

(27) Yin, W.-J.; Shi, T.; Yan, Y. Unique Properties of Halide Perovskites as Possible Origins of the Superior Solar Cell Performance. Adv. Mater. 2014, 26, 4653-4658.

(28) He, Y.; Galli, G. Perovskites for Solar Thermoelectric Applications: a First Principle Study of $\mathrm{CH}_{3} \mathrm{NH}_{3} \mathrm{AI}_{3}(\mathrm{~A}=\mathrm{Pb}$ and Sn). Chem. Mater. 2014, 26, 5394-5400.

(29) Geng, W.; Zhang, L.; Zhang, Y.-N.; Lau, W.-M.; Liu, L.-M. FirstPrinciples Study of Lead Iodide Perovskite Tetragonal and Orthorhombic Phases for Photovoltaics. J. Phys. Chem. C 2014, 118, 19565-19571.

(30) Brivio, F.; Butler, K. T.; Walsh, A.; van Schilfgaarde, M. Relativistic Quasiparticle Self-Consistent Electronic Structure of Hybrid Halide Perovskite Photovoltaic Absorbers. Phys. Rev. B: Condens. Matter Mater. Phys. 2014, 89, 155204.

(31) Filip, M. R.; Verdi, C.; Giustino, F. GW Band Structures and Carrier Effective Masses of $\mathrm{CH}_{3} \mathrm{NH}_{3} \mathrm{PbI}_{3}$ and Hypothetical Perovskites of the Type $\mathrm{APbI}_{3}: \mathrm{A}=\mathrm{NH}_{4}, \mathrm{PH}_{4}, \mathrm{AsH}_{4}$, and $\mathrm{SbH}_{4}$. J. Phys. Chem. C 2015, 119, 25209-25219.

(32) Miyata, A.; Mitioglu, A.; Plochocka, P.; Portugall, O.; Wang, J. T.-W.; Stranks, S. D.; Snaith, H. J.; Nicholas, R. J. Direct Measurement of the Exciton Binding Energy and Effective masses for Charge Carriers in Organic-iIorganic Tri-Halide Perovskites. Nat. Phys. 2015, $11,582-587$.
(33) Stoumpos, C. C.; Malliakas, C. D.; Kanatzidis, M. G. Semiconducting Tin and Lead Iodide Perovskites with Organic Cations: Phase Transitions, High Mobilities, and Near-Infrared Photoluminescent Properties. Inorg. Chem. 2013, 52, 9019-9038.

(34) Berhe, T. A.; Su, W.-N.; Chen, C.-H.; Pan, C.-J.; Cheng, J.-H.; Chen, H.-M.; Tsai, M.-C.; Chen, L.-Y.; Dubale, A. A.; Hwang, B.-J. Organometal Halide Perovskite Solar Cells: Degradation and Stability. Energy Environ. Sci. 2016, 9, 323-356.

(35) Swarnkar, A.; Marshall, A. R.; Sanehira, E. M.; Chernomordik, B. D.; Moore, D. T.; Christians, J. A.; Chakrabarti, T.; Luther, J. M. Quantum Dot-Induced Phase Stabilization of $\alpha-\mathrm{CsPbI}_{3}$ Perovskite for High-Efficiency Photovoltaics. Science 2016, 354, 92-95.

(36) Zhang, D.; Eaton, S. W.; Yu, Y.; Dou, L.; Yang, P. SolutionPhase Synthesis of Cesium Lead Halide Perovskite Nanowires. J. Am. Chem. Soc. 2015, 137, 9230-9233.

(37) Protesescu, L.; Yakunin, S.; Bodnarchuk, M. I.; Krieg, F.; Caputo, R.; Hendon, C. H.; Yang, R. X.; Walsh, A.; Kovalenko, M. V. Nanocrystals of Cesium Lead Halide Perovskites $\left(\mathrm{CsPbX}_{3}, \mathrm{X}=\mathrm{Cl}, \mathrm{Br}\right.$, and I): Novel Optoelectronic Materials Showing Bright Emission with Wide Color Gamut. Nano Lett. 2015, 15, 3692-3696.

(38) Saliba, M.; Matsui, T.; Domanski, K.; Seo, J.-Y.; Ummadisingu, A.; Zakeeruddin, S. M.; Correa-Baena, J.-P.; Tress, W. R.; Abate, A.; Hagfeldt, A.; et al. Incorporation of Rubidium Cations into Perovskite Solar Cells Improves Photovoltaic Performance. Science 2016, 354, 206-209.

(39) Abdi-Jalebi, M.; Dar, M. I.; Sadhanala, A.; Senanayak, S. P.; Franckevičius, M.; Arora, N.; Hu, Y.; Nazeeruddin, M. K.; Zakeeruddin, S. M.; Grätzel, M.; Friend, R. H. Impact of Monovalent Cation Halide Additives on the Structural and Optoelectronic Properties of $\mathrm{CH}_{3} \mathrm{NH}_{3} \mathrm{PbI}_{3}$ Perovskite. Adv. Energy Mater. 2016, 6, 1502472.

(40) Mosconi, E.; Quarti, C.; Ivanovska, T.; Ruani, G.; De Angelis, F. Structural and Electronic Properties of Organo-Halide Lead Perovskites: a Combined IR-Spectroscopy and Ab Initio Molecular Dynamics Investigation. Phys. Chem. Chem. Phys. 2014, 16, 1613716144.

(41) Mattoni, A.; Filippetti, A.; Saba, M.; Delugas, P. Methylammonium Rotational Dynamics in Lead Halide Perovskite by Classical Molecular Dynamics: the Role of Temperature. J. Phys. Chem. C 2015, 119, 17421-17428.

(42) Meloni, S.; Moehl, T.; Tress, W.; Franckevičius, M.; Saliba, M.; Lee, Y. H.; Gao, P.; Nazeeruddin, M. K.; Zakeeruddin, S. M.; Rothlisberger, U.; et al. Ionic Polarization-Induced Current-Voltage Hysteresis in $\mathrm{CH}_{3} \mathrm{NH}_{3} \mathrm{PbX}_{3}$ Perovskite Solar Cells. Nat. Commun. 2016, 7, 10334

(43) Leguy, A. M. A.; Frost, J. M.; McMahon, A. P.; Sakai, V. G.; Kochelmann, W.; Law, C.; Li, X.; Foglia, F.; Walsh, A.; O’regan, B. C. The Dynamics of Methylammonium Ions in Hybrid Organic-Inorganic Perovskite Solar Cells. Nat. Commun. 2015, 6, 7124.

(44) Chen, T.; Foley, B. J.; Ipek, B.; Tyagi, M.; Copley, J. R.; Brown, C. M.; Choi, J. J.; Lee, S.-H. Rotational Dynamics of Organic Cations in the $\mathrm{CH}_{3} \mathrm{NH}_{3} \mathrm{PbI}_{3}$ Perovskite. Phys. Chem. Chem. Phys. 2015, 17, 31278-31286.

(45) Brivio, F.; Walker, A. B.; Walsh, A. Structural and Electronic Properties of Hybrid Perovskites for High-Efficiency Thin-Film Photovoltaics from First-Principles. APL Mater. 2013, 1, 04211.

(46) Yin, W.-J.; Yang, J.-H.; Kang, J.; Yan, Y.; Wei, S.-H. Halide Perovskite Materials for Solar Cells: a Theoretical Review. J. Mater. Chem. A 2015, 3, 8926-8942.

(47) Harrison, W. A. Solid State Theory; Dover Books on Physics; Courier Corporation: North Chelmsford, MA, 1970.

(48) Ashcroft, N. W.; Mermin, N. D. Solid State Physics; Harcourt College Publishers: Fort Worth, TX, 1976.

(49) Zheng, F.; Tan, L. Z.; Liu, S.; Rappe, A. M. Rashba Spin-Orbit Coupling Enhanced Carrier Lifetime in $\mathrm{CH}_{3} \mathrm{NH}_{3} \mathrm{PbI}_{3}$. Nano Lett. 2015, 15, 7794-7800.

(50) Kepenekian, M.; Robles, R.; Katan, C.; Sapori, D.; Pedesseau, L.; Even, J. Rashba and Dresselhaus Effects in Hybrid Organic-Inorganic 
Perovskites: From Basics to Devices. ACS Nano 2015, 9, 11557-

11567.

(51) Feng, J.; Xiao, B. Effective Masses and Electronic and Optical Properties of Nontoxic $\mathrm{MASnX}_{3}(\mathrm{X}=\mathrm{Cl}, \mathrm{Br}$, and I) Perovskite Structures as Solar Cell Absorber: A Theoretical Study Using HSE06.

J. Phys. Chem. C 2014, 118, 19655-19660.

(52) Perdew, J. P.; Burke, K.; Wang, Y. Generalized Gradient Approximation for the Exchange-Correlation Hole of a Many-Electron System. Phys. Rev. B: Condens. Matter Mater. Phys. 1996, 54, 16533.

(53) Giannozzi, P.; Baroni, S.; Bonini, N.; Calandra, M.; Car, R.; Cavazzoni, C.; Ceresoli, D.; Chiarotti, G. L.; Cococcioni, M.; Dabo, I.; et al. QUANTUM ESPRESSO: A Modular and Open-Source Software Project for Quantum Simulations of Materials. J. Phys.: Condens. Matter 2009, 21, 395502.

(54) Monkhorst, H. J.; Pack, J. D. Special Points for Brillouin-Zone Integrations. Phys. Rev. B 1976, 13, 5188.

(55) MATLAB, version 7.10.0 (R2010a); The MathWorks Inc.: Natick, MA, 2010.

(56) Wang, Y.; Gould, T.; Dobson, J. F.; Zhang, H.; Yang, H.; Yao, X.; Zhao, H. Density Functional Theory Analysis of Structural and Electronic Properties of Orthorhombic Perovskite $\mathrm{CH}_{3} \mathrm{NH}_{3} \mathrm{PbI}_{3}$. Phys. Chem. Chem. Phys. 2014, 16, 1424-1429.

(57) Even, J.; Pedesseau, L.; Katan, C. Comment on "Density Functional Theory Analysis of Structural and Electronic Properties of Orthorhombic Perovskite $\mathrm{CH}_{3} \mathrm{NH}_{3} \mathrm{PbI}_{3}$ ” by Y. Wang et al., Phys. Chem. Chem. Phys., 2014, 16, 1424-1429. Phys. Chem. Chem. Phys. 2014, 16, 8697-8698.

(58) Grimme, S. Semiempirical GGA-type Density Functional Constructed with a Long-Range Dispersion correction. J. Comput. Chem. 2006, 27, 1787-1799.

(59) Dion, M.; Rydberg, H.; Schröder, E.; Langreth, D. C.; Lundqvist, B. I. Van der Waals Density Functional for General Geometries. Phys. Rev. Lett. 2004, 92, 246401.

(60) Lee, K.; Murray, É. D.; Kong, L.; Lundqvist, B. I.; Langreth, D. C. Higher-Accuracy van der Waals Density Functional. Phys. Rev. B: Condens. Matter Mater. Phys. 2010, 82, 081101.

(61) Walsh, A.; Scanlon, D. O.; Chen, S.; Gong, X.; Wei, S.-H. SelfRegulation Mechanism for Charged Point Defects in Hybrid Halide Perovskites. Angew. Chem. 2015, 127, 1811-1814.

(62) Yin, W.-J.; Shi, T.; Yan, Y. Unusual Defect Physics in $\mathrm{CH}_{3} \mathrm{NH}_{3} \mathrm{PbI}_{3}$ Perovskite Solar Cell Absorber. Appl. Phys. Lett. 2014, 104, 063903.

(63) Wen, X.; Feng, Y.; Huang, S.; Huang, F.; Cheng, Y.-B.; Green, M.; Ho-Baillie, A. Defect Trapping States and Charge Carrier Recombination in Organic-inorganic Halide Perovskites. J. Mater. Chem. C 2016, 4, 793.

(64) Shi, D.; Adinolfi, V.; Comin, R.; Yuan, M.; Alarousu, E.; Buin, A.; Chen, Y.; Hoogland, S.; Rothenberger, A.; Katsiev, K.; et al. Low Trap-State Density and Long Carrier Diffusion in Organolead Trihalide Perovskite Single Crystals. Science 2015, 347, 519-522.

(65) Kumar, M. H.; Dharani, S.; Leong, W. L.; Boix, P. P.; Prabhakar, R. R.; Baikie, T.; Shi, C.; Ding, H.; Ramesh, R.; Asta, M.; et al. LeadFree Halide Perovskite Solar Cells with High Photocurrents Realized Through Vacancy Modulation. Adv. Mater. 2014, 26, 7122-7127.

(66) Kim, J.; Lee, S.-H.; Lee, J. H.; Hong, K.-H. The Role of Intrinsic Defects in Methylammonium Lead Iodide Perovskite. J. Phys. Chem. Lett. 2014, 5, 1312-1317.

(67) Lany, S.; Zunger, A. Assessment of Correction Methods for the Band-Gap Problem and for Finite-Size Effects in Supercell Defect Calculations: Case Studies for $\mathrm{ZnO}$ and GaAs. Phys. Rev. B: Condens. Matter Mater. Phys. 2008, 78, 235104.

(68) Menéndez-Proupin, E.; Palacios, P.; Wahnón, P.; Conesa, J. SelfConsistent Relativistic Band Structure of the $\mathrm{CH}_{3} \mathrm{NH}_{3} \mathrm{PbI}_{3}$ Perovskite. Phys. Rev. B: Condens. Matter Mater. Phys. 2014, 90, 045207.

(69) Mosconi, E.; Meggiolaro, D.; Snaith, H. J.; Stranks, S. D.; De Angelis, F. Light-Induced Annihilation of Frenkel Defects in OrganoLead Halide Perovskites. Energy Environ. Sci. 2016, 9, 3180-3187.

(70) Ball, J. M.; Petrozza, A. Defects in Perovskite-Halides and Their Effects in Solar Cells. Nat. Energy 2016, 1, 16149. 